\title{
11. FORAMINIFERS AND COARSE DETRITUS FROM FIVE DEEP-WATER SITES, DEEP SEA DRILLING PROJECT LEG 86, NORTHWEST PACIFIC ${ }^{1}$
}

\author{
Anthony D'Agostino, ARCO Exploration Company²
}

\begin{abstract}
Five sites of Leg 86 of the Deep Sea Drilling Project were drilled in water greater than $5000 \mathrm{~m}$ deep in the northwest Pacific. The objectives of the coring program at these sites included sampling Cenozoic pelagic sediments, in an effort to define paleoceanographic patterns and boundaries, and assessing the nature of the red clay/chert boundary which is seismically mappable. The biostratigraphy of foraminifers contributes to these objectives in only four short intervals. Cenozoic pelagic sediments contained rare, small, moderately preserved assemblages of planktonic foraminifers. Calcareous sediments below $55.3 \mathrm{~m}$ sub-bottom at Site 576 are turbidites of Cretaceous age containing Santonian/Campanian foraminifers. A small assemblage of early Eocene age (P9-P11) was found at $163.28 \mathrm{~m}$ at Site 578, corroborating a similar determination based on ichthyoliths. Foraminifers in calcareous sediment immediately above the chert at $176.44 \mathrm{~m}$ sub-bottom at Site 578 indicate a Campanian minimum for the age of the chert horizon at that location. At Site 580, a modest assemblage of late Pleistocene (N23) age was recovered above $41.29 \mathrm{~m}$ sub-bottom and an early Pleistocene (N22) assemblage found in one sample at $44.89 \mathrm{~m}$ sub-bottom. Samples from Sites 579 and 581 were barren of foraminifers.

Examination of the washed residue for foraminifers disclosed abundant volcanic glass, Mn-oxide nodules and tubes, and eolian silt in the greenish and red pelagic clays. Biosiliceous debris and ichthyoliths are also common to abundant. Middle Miocene deposits at Site 581 contain ice-rafted basalt and andesite pebbles.
\end{abstract}

\section{INTRODUCTION}

Five deep-water sites were planned for Leg 86 (Fig. 1). The site objectives were to study the nature and rate of Cenozoic sedimentation and the chert/clay horizon. The Cenozoic sediments were expected to be predominantly biosiliceous, so the importance of calcareous fossil groups was expected to be minimal. The seismically mappable chert layer was, however, thought to be associated with calcareous deposits and foraminifers were expected to be significant in dating this horizon.

The biostratigraphic utility of foraminifers was indeed limited by rare occurrences of planktonic or benthic species in recovered Cenozoic sediments. One Cretaceous calcareous turbidite (Site 576) was penetrated above the chert, and Campanian calcareous sediment was recovered at Site 580, just above the chert. Besides foraminifers, sample examination uncovered manganese-oxide replacement of foraminifers and middle Miocene ice-rafted detritus. Results of the study of Cretaceous and Cenozoic calcareous sediment recovered from Site 577 on the Shatsky Rise will be published elsewhere.

Cored sediment is divided into lithologic units on the basis of color, smear-slide, textural, and compositional data. Three units were designated at Site 576 (see Site 576 chapter, this volume). Lithologic Unit I (0-55 m subbottom) consisted of yellowish brown to brown pelagic clay above $28 \mathrm{~m}$ sub-bottom (Subunit IA) and dark brown "slick" clay below (Subunit IB). Lithologic Unit II is an interbedded dark brown pelagic clay and pale brown nannofossil ooze. Lithologic Unit III (76 m sub-bottom) is

\footnotetext{
I Heath, G. R., Burckle, L. H., et al., Init. Repts. DSDP, 86:Washington (U.S. Govt. Printing Office).

2 Address: ARCO Exploration Company, P.O. Box 1346, Houston, TX 77056.
}

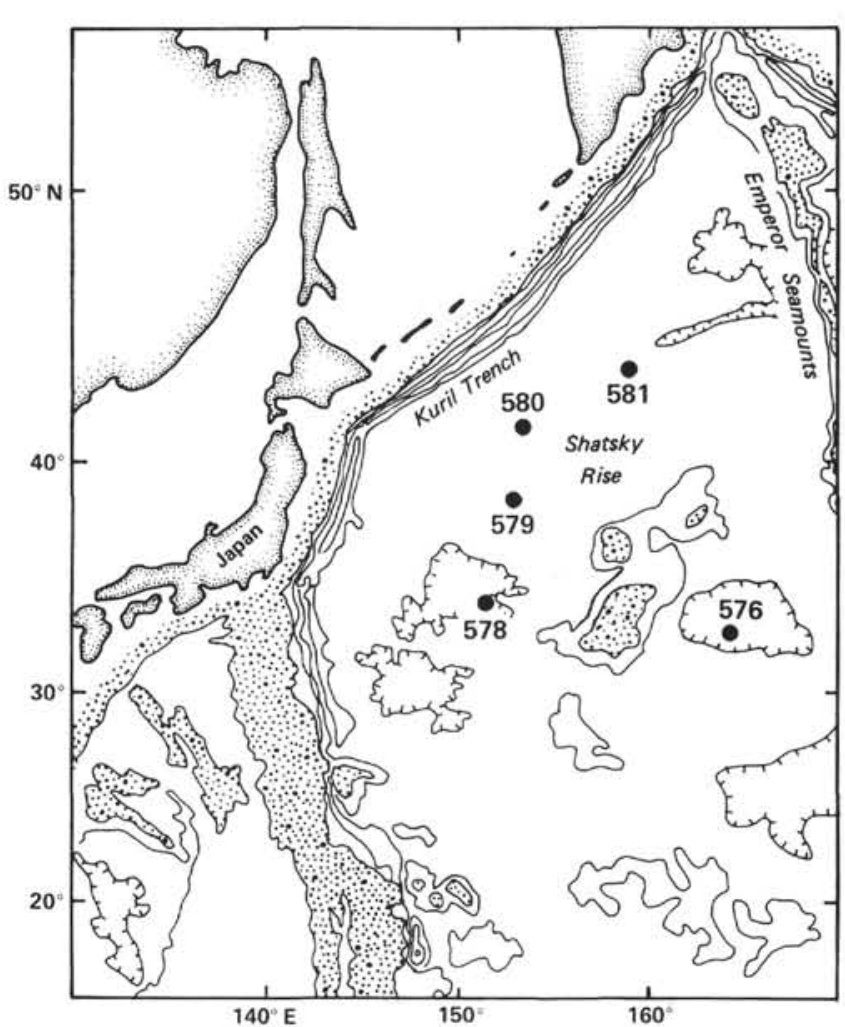

Figure 1. Map showing location of the five deep-water sites: 576, 578, 579,580 , and 581 . Areas shallower than $4 \mathrm{~km}$ stippled, $5-\mathrm{km}$ contour plain, $6-\mathrm{km}$ contour hachured.

chert and porcellanite. Four units were designated at Site 578 (see Site 578 chapter, this volume). Lithologic Unit I ( $0-76.6 \mathrm{~m}$ sub-bottom) is a gray to olive gray clay and siliceous clay, Lithologic Unit II (76.6-124.5 m sub- 
bottom) is a yellowish-brown to brown clay containing minor amounts of siliceous microfossils. Lithologic Unit III (124.5-176 m sub-bottom) is a dark brown pelagic clay. It contains $90 \%$ or more clay and biogenic silica is virtually absent. Lithologic Unit IV (176.0-176.8 m subbottom) consists of interlaminated brown, yellow, and gray clays over chert. One major unit was designated at Site 579 (see Site 579 chapter, this volume). Lithologic Unit I was subdivided into three subunits (IA, 0-60 m sub-bottom; IB, 60-102.5 m sub-bottom; IC, 102.5$149.5 \mathrm{~m}$ sub-bottom) based on the abundance of biogenic silica, quartz, and clay. One lithologic unit was designated at Site 580 (see Site 580 chapter, this volume). Unit $\mathrm{I}$ is a siliceous clay subdivided into five subunits (IA, 0-60.3 m sub-bottom; IB, 60.3-79.3 m sub-bottom; IC, 79.3-117.3 m sub-bottom; ID, 117.3-136.3 m subbottom; IE, 136.3-155.3 m sub-bottom) based on the content of clay, quartz, biogenic silica, and clay-sized carbonate. Four units were designated at Site 581 (see Site 581 chapter, this volume). Lithologic Unit I (0$244.8 \mathrm{~m}$ sub-bottom) is a gray green or yellowish brown biosiliceous clay. Unit II (244.8-276.6 m sub-bottom) is a dark brown "slick" pelagic clay. Unit III (276.7-343 m sub-bottom) is chert and porcellanite. Unit IV (343$352.5 \mathrm{~m}$ sub-bottom) is aphyric basalt.

\section{METHODS}

Samples were disaggregated in distilled water and occasionally hydrogen peroxide or Calgon was used to aid in dispersal of clays. The sediment was then washed over 149 - and $63-\mu \mathrm{m}$ sieves. The dried residues then placed in separate containers for each size fraction and examined with a binocular microscope.

\section{RESULTS}

\section{Site 576: Holes 576 and 576B}

Site 576 is located east of the Shatsky Rise in $6220 \mathrm{~m}$ of water. The dark brown pelagic clay of Unit I (0-55 m sub-bottom) (Fig. 2) contains no planktonic foraminifers and only rare specimens of Reophax nodulosus and $R$. difflugiformis. Mn-oxide replacement of foraminifers was also noted in the sediments of Unit I. This phenomenon was also reported by Thompson (1980) in sediments from the Japan Trench.

Sequences of massive, cross-bedded and normally graded units exhibiting sharp and gradational contacts are common in sediments deposited by turbidity flows. The calcareous sediments of Unit II exhibit these features. The calcareous oozes are also separated by greenish pelagic clay containing Late Cretaceous ichthyoliths (see Site 576 chapter, this volume). Interbedded pelagic sediments characteristically overlie turbidity flows in a deep-sea environment. The calcareous units containing Santonian-Campanian foraminifers can be correlated between the two holes and a sequence of 23 turbidity flows can be recognized (Fig. 3). The reworked nature of the sediments is also indicated by the wide age range indicated by the foraminifers. The specimens found are typical of Late Cretaceous foraminiferal assemblages as reported in the U.S. Gulf Coast (Pessagno, 1967), New Jersey (Olsson, 1960; Petters, 1977), Shatsky Rise (Ca- ron, 1975; Hofker, 1978), the Pacific west of the Shatsky Rise (Krasheninnikov and Hoskins, 1973), the western interior seaway of the U.S. (Frerichs and Dring, 1981), northern California (Trujillo, 1960), and Vancouver, B.C. (McGugan, 1982).

All sub-bottom depths given here and in Appendix $\mathrm{B}$ are adjusted according to the factors determined by Ross Heath. Details and techniques used for the adjustments are given in the Site 576 chapter (this volume). Appendix A contains lists of species found in the turbidites and clays recovered at Site 576. Appendix B contains detailed sample information for the two holes.

\section{Site 578: Hole 578}

Site 578 was the southernmost site in a northerly transect on Leg 86. It is located west of the Shatsky Rise in $6020 \mathrm{~m}$ of water. Foraminifers are rare in the late Pleistocene siliceous clay of Lithologic Subunit IA and in the pelagic clay of Lithologic Unit II. A small assemblage containing two species can be used to date a portion of Lithologic Unit III. A small but diverse assemblage was found just above the chert in Lithologic Unit IV. Sample $578-1-3,80-82 \mathrm{~cm}$ (4.79 m sub-bottom) contained rare specimens of Reophax difflugiformis and Chilostomella sp. Sample 578-12,CC (109.29 m sub-bottom) contained two specimens of Massilina tenuis. Sample 57818 , CC $(18-20 \mathrm{~cm})(163.28 \mathrm{~m}$ sub-bottom) contained rare specimens of Globorotalia spinulosa and G. broederman$n i$. These indicate an early Eocene age, but the precise zonation is indeterminate. Their ranges span P9-P11 (the Globorotalia pentacamerata Zone to the Globigerinatheka subconglobata Zone; Stainforth et al., 1975). Samples 578-20-1, 64-66 cm and 578-20,CC (176.45 m, $176.8 \mathrm{~m}$ sub-bottom) contained abundant specimens of Globotruncana fornicata; common G. tricarinata, G. lapparenti, Pseudotextularia elegans, and Heterohelix striata; and rare to few Globigerinelloides asperus, $G$. prairiehillensis, Hedbergella planispira, Globotruncana arca, G. stuartiformis, and Rugoglobigerina rugosa. A Late Cretaceous Campanian age is indicated in the Globotruncana fornicata-stuartiformis Zone of Pessagno (1967).

Appendix B contains detailed information on the particles observed in these samples including $\mathrm{Mn}$ nodules and ichthyoliths.

\section{Site 579: Holes 579, 579A}

This site was the second in the north-south paleoceanographic transect and is located in $5746 \mathrm{~m}$ of water. No foraminifers were recovered. Appendix B lists observations on the detritus found in the samples examined.

\section{Site 580: Hole 580}

Site 580 is located in $5385 \mathrm{~m}$ of water and is the third in the series of sites in the north-south transect on Leg 86. Foraminifers were found only in the Pleistocene siliceous clay of lithologic Subunit IA (above $44.9 \mathrm{~m}$ subbottom). Planktonic and benthic species are typically rare (Table 1). Preservation is moderate to good, with no evident abrasion or breakage, and dissolution effects are light to moderate. The presence of these specimens in the 


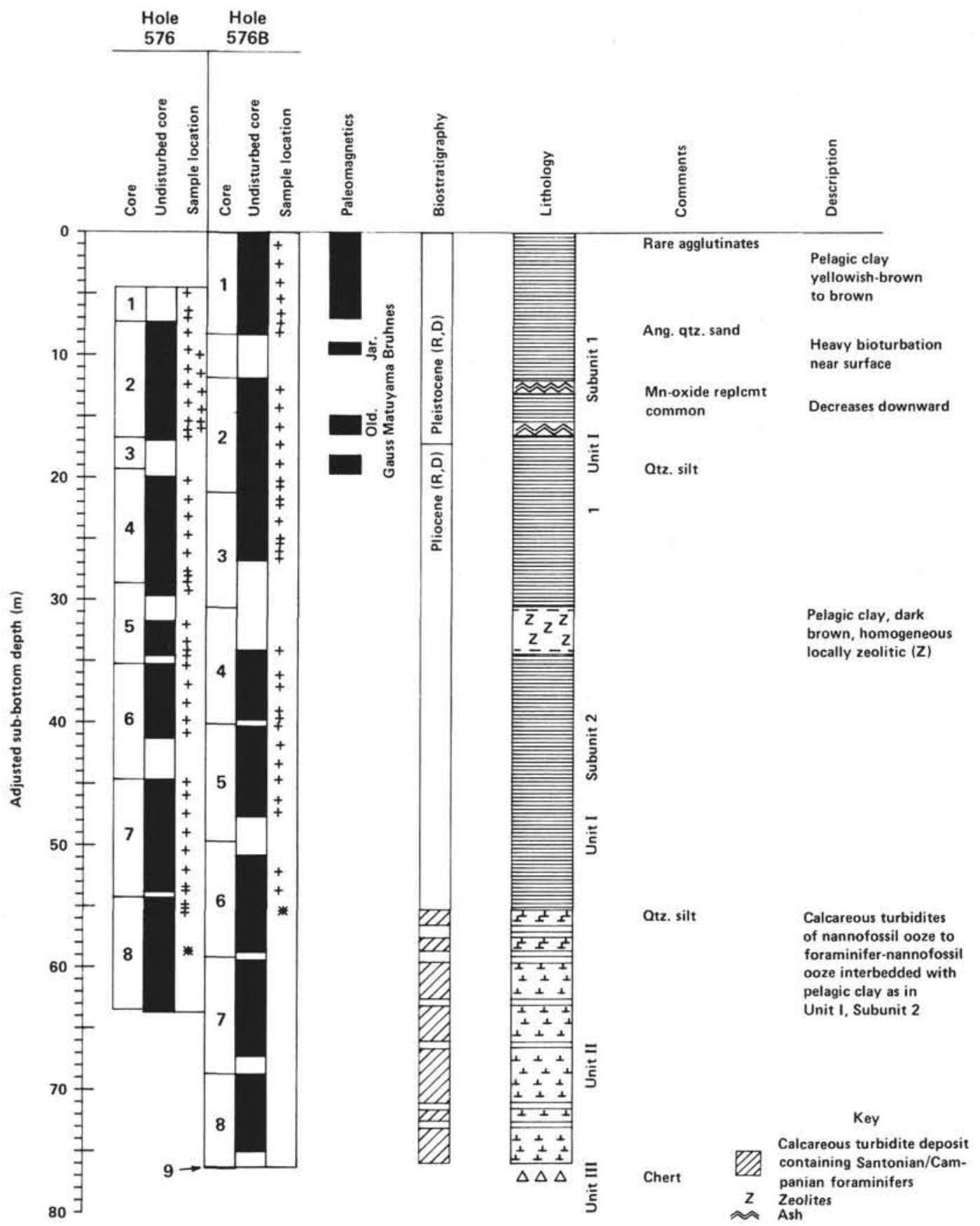

Figure 2. Sample location, paleomagnetics, biostratigraphy, and core description for Holes 576 and 576B. For information on samples below asterisks, see Figure 3.

biosiliceous clay indicates that sedimentation rate alone does not control the preservation of calcareous fossils since the rate apparently has not changed at that site since the mid-Pliocene. Appendix B contains detailed sample information for Site 580 .

\section{Site 581: Hole 581}

This hole was the last and most northerly location drilled on Leg 86 . The water depth of $5486 \mathrm{~m}$ is reflected by the total absence of foraminifers in recovered sediment. Two samples analyzed from Core 581-9 (middle Miocene, see Site 581 chapter, this volume) contain rounded basalt and andesite pebbles indicative of ice-rafted de- tritus deposition at this location (Duennebier, Stephen, Gettrust et al., in press).

\section{SUMMARY}

The biostratigraphic utility of foraminifers was limited at the five deep-water sites visited in the northwest Pacific during DSDP Leg 86. At Site 576, foraminifers are rare to absent except in the calcareous sediment below $55.3 \mathrm{~m}$ sub-bottom. Because of the turbiditic nature of the deposits, foraminifers could not be used to date them. At Sites 579, 580, and 581 foraminifers were either rare or absent and merely supplemented the detailed siliceous biostratigraphy provided by diatoms, ra- 
diolarians, and silicoflagellates. The stated objective of dating the chert/clay boundary was accomplished only at Site 578 , where a minimum Campanian age can be assigned to the chert. Figure 4 summarizes the foraminiferal biostratigraphy of the deep-water sites of Leg 86 .

\section{ACKNOWLEDGMENT}

The author wishes to thank ARCO Exploration Co. and Mssrs. G. B. Martin and K. Geitzenaur for the leave of absence required to take part in Leg 86. Gerta Keller reviewed this manuscript.

\section{REFERENCES}

Blow, W. H., 1969. Late middle Eocene to Recent planktonic biostratigraphy. Proc. Int. Conf. Planktonic Microfossils, Ist, Geneva, $1967,1: 199-422$.

Caron, M., 1975. Late Cretaceous planktonic foraminifera from the northwestern Pacific, Leg 32 of the Deep Sea Drilling Project. In Larson, R. L., Moberly, R., Init. Repts. DSDP, 32: Washington (U.S. Govt. Printing Office), 719-724.

Duennebier, F. K., Stephen, R., Gettrust, J. F., et al., in press. Site 581. In Duennebier, F. K., Stephen, R., Gettrust, J. F., et al., Init. Repts. DSDP, 88/91: Washington (U.S. Govt. Printing Office).

Frerichs, W. E., and Dring, N. B., 1981. Planktonic foraminifera from the Smoky Hill Shale of West Central Kansas. J. Foraminiferal Res., 11(1):47-69.

Hofker, J., 1978. Analysis of a large succession of samples through the upper Maestrichtian and the lower Tertiary of drill Hole 47.2, Shatsky Rise, Pacific, Deep Sea Drilling Project. J. Foraminiferal Res., 8(1):46-75.

Krasheninnikov, V., and Hoskins, R. H., 1973. Late Cretaceous, Paleogene, and Neogene planktonic foraminifera. In Heezen, B. C., MacGregor, I. D., et al., Init. Repts. DSDP, 20: Washington (U.S. Govt. Printing Office), 105-203.

McGugan, A., 1982. Upper Cretaceous (Campanian and Maestrichtian) foraminifera from the upper Lambert and Northumberland formations, Gulf Islands, British Columbia, Canada. Micropaleontology, 28(4):399-430.

Olsson, R. K., 1960. Foraminifera of the Latest Cretaceous and earliest Tertiary Age in the New Jersey Coastal Plain. J. Paleontol., 34(1):1-58.

Pessagno, E. A., 1967. Upper Cretaceous planktonic foraminifera from the Western Gulf Coastal Plain. Paleontographica Americana, $\mathrm{V}(37): 243-445$

Petters, S. W., 1977. Upper Cretaceous planktonic foraminifera from the subsurface of the Atlantic Coastal Plain of New Jersey. J. Foraminiferal Res., 7(3):165-187.

Stainforth, R. M., Lamb, J. L., Luterbacher, H., Beard, J. H., and Jeffords, R. M., 1975. Cenozoic planktonic foraminiferal zonation and characteristics of index forms. Univ. Kans. Paleontol. Contrib. Pap., 62.

Thompson, P. R., 1980. Foraminifers from Deep Sea Drilling Project Sites 434, 435, and 436, Japan Trench. In Scientific Party, Init. Repts. DSDP, 56, 57, Pt. 2: Washington (U.S. Govt. Printing Office), $775-808$.

Trujillo, E. F., 1960. Upper Cretaceous foraminifera near Redding, Shasta County, California. J. Paleontol., 34:290-346.

Date of Initial Receipt: 25 April 1984

Date of Acceptance: 27 July 1984

\section{APPENDIX A}

Listing of Species, Holes 576 and 576B

Species found in Hole 576 turbidites

Ammodiscus asperellus (d'Orbigny)

Aragonia velascoensis (Cushman)

Archaeoglobigerina blowi Pessagno

A. tradinghousensis (Pessagno)

Bolivinoides sp.

Bulimina quadrata Plummer

Dentalina colei Cushman \& Dusenbury

D. peracuta (Reuss)

D. wilcoxensis Cushman

Dentalina sp.

Eponides bandyi Trujillo
E. simplex (White)

Fissurina sp.

Globigerinelloides asperus (Ehrenberg)

G. prairiehillensis Pessagno

Globotruncana angusticarinata (Gandolfi)

G. bulloides Volger

G. elevata (Brotzen)

G. lapparenti Brotzen

G. stuartiformis Dalbiez

Gyroidina tsablensis McGugan

Haplophragmoides multicameras Krasheninnikov

$H$. pervagatus Krasheninnikov

Hastigerinoides watersi (Cushman)

Hedbergella planispira (Tappan)

Heterohelix globulosa (Ehrenberg)

H. pulchra (Brotzen)

H. reussi (Cushman)

H. striata (Ehrenberg)

Lagena gracilis (d'Orbigny)

Melonis affinis Reuss

Nodosaria tenuicosta Reuss

Ostracod fragments

Paratrochamminoides intracatus Krasheninnikov

P. semipellucidus Krasheninnikov

$P$. vitreus Krasheninnikov

Praecystammina globigerinaeformis Krasheninnikov

Pseudotextularia elegans (Rzehak)

Reophax nodulosus Cushman

Reophax sp.

Schackoina multispinata (Cushman \& Wickenden)

Stilostomella midwayensis (Cushman \& Todd)

Species found in Hole 576B

Aragonia velascoensis (Cushman)

Archaeoglobigerina blowi Pessagno

A. bosquensis Pessagno

A. tradinghousensis (Pessagno)

Bulimina sp.

Dentalina colei Cushman \& Dusenbury

D. peracuta (Reuss)

D. semipellucidus Krasheninnikov

Dentalina wilcoxensis Cushman

Dentalina sp.

Eponides bandyi Trujillo

E. simplex (White)

Fissurina sp.

Glandulina pygmaea Reuss

Globigerinelloides asperus (Ehrenberg)

G. prairiehillensis Pessagno

Globotruncana angusticarinata (Gandolfi)

G. bulloides Volger

G. calcarata Cushman

G. elevata (Brotzen)

G. lapparenti Brotzen

G. stuartiformis Dalbiez

Gyroidina tsablensis McGugan

Haplophragmoides biumbilicalis Krasheninnikov

$H$. multicamerus Krasheninnikov

$H$. pervagatus Krasheninnikov

Hastigerinoides watersi (Cushman)

Hedbergella planispira (Tappan)

Heterohelix globulosa (Ehrenberg)

H. pulchra (Brotzen)

H. reussi (Cushman)

H. striata (Ehrenberg)

Lagena gracilis (d'Orbigny)

Lenticulina sp.

Melonis affinis Reuss

Mn-oxide replacements

Nodosaria tenuicosta Reuss

Osangularia sp.

Paratrochamminoides intracatus Krasheninnikov

$P$. vitreus Krasheninnikov

Praecystammina globigerinaeformis Krasheninnikov

Pseudotextularia elegans (Rzehak)

Schackoina multispinata (Cushman \& Wickendam)

Stilostomella midwayensis (Cushman \& Todd) 


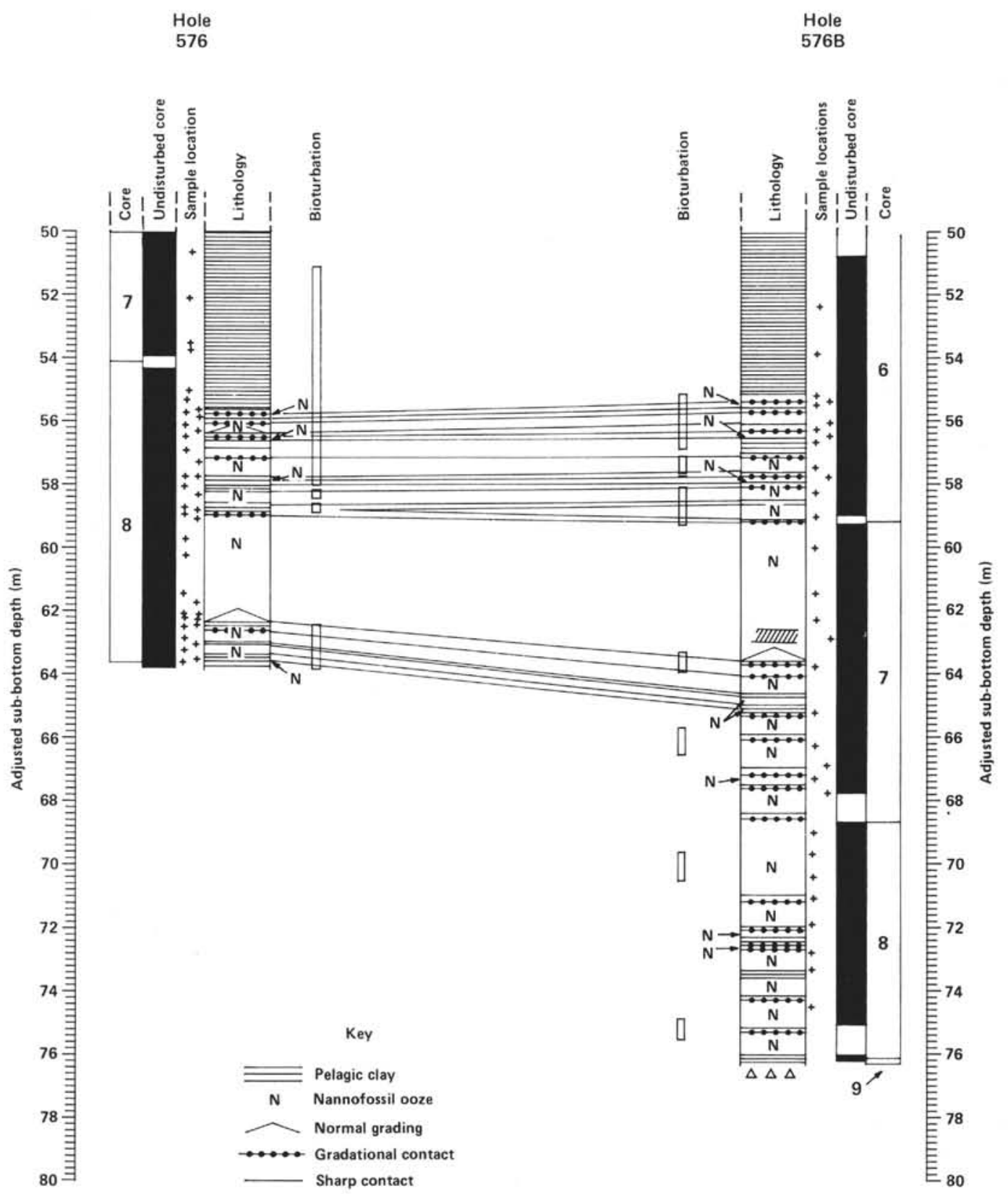

Figure 3. Sample location, lithology, bioturbation, and correlation of calcareous turbidites in Holes 576 and 576B.

Table 1. Occurrence and abundance of foraminifers in samples from Hole 580.

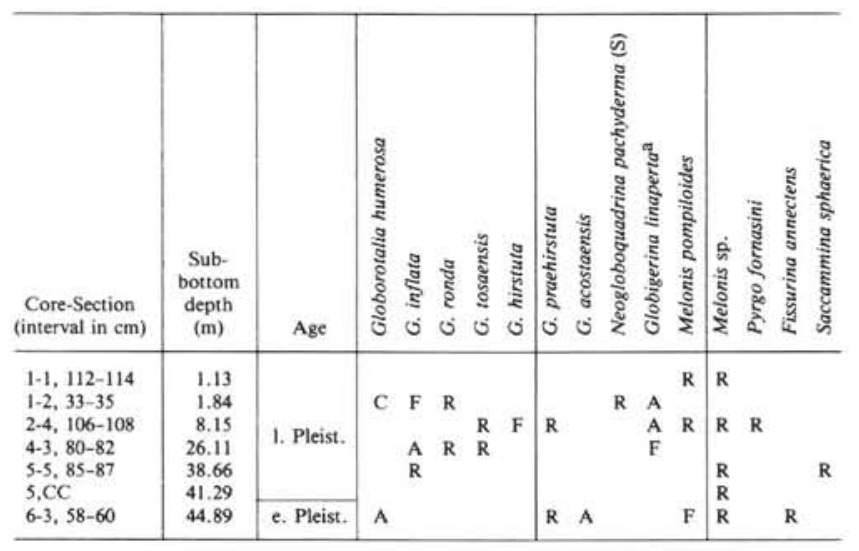

${ }^{a}$ Reworked. 


\section{A. D'AGostino}

\begin{tabular}{|c|c|c|c|c|c|c|}
\hline \multirow{2}{*}{ Age } & Zone & Zone & Hole 576 & Hole 576B & Hole 578 & Hole 580 \\
\hline & Stainforth et al. (1975) & Blow (1969) & Sub-bottom sample & Sub-bottom sample & Sub-bottom sample & Sub-bottom sample \\
\hline 兽 & \multirow{2}{*}{$\begin{array}{l}\text { Globorotalia } \\
\text { truncatulinoides } \\
\text { Zone }\end{array}$} & & & & & $\begin{array}{l}41.29 \mathrm{~m} \quad 5, \mathrm{CC} \\
\text { (and above) }\end{array}$ \\
\hline 㪰高 & & N22 & & & & $\begin{array}{l}44.89 \mathrm{~m} \\
6-3,58-60 \mathrm{~cm} \\
\text { (only) }\end{array}$ \\
\hline 늘 & $\begin{array}{c}\text { Globigerinatheka } \\
\text { subconglobata Zone } \\
\text { to } \\
\text { Globorotalia } \\
\text { pentacamerata Zone }\end{array}$ & P11 to P9 & & 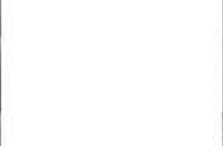 & $\begin{array}{l}163.28 \mathrm{~m} \\
18, \mathrm{CC} \\
(18-20 \mathrm{~cm}) \\
\text { (only) }\end{array}$ & \\
\hline \multirow{3}{*}{ 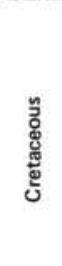 } & \multirow[t]{2}{*}{$\begin{array}{l}\text { Cretaceous by } \\
\text { ichthyolith }\end{array}$} & \multirow[b]{2}{*}{ Maest.? } & $\begin{array}{l}\text { Below } \overline{8} \cdot \overline{1}, \overline{135}=- \\
137 \mathrm{~cm} 55.34 \mathrm{~m}\end{array}$ & \multirow{2}{*}{$\begin{array}{l}\text { Below } \overline{6} \cdot \overline{5}, \overline{24}-\overline{2} 6 \mathrm{~cm} \\
55.83 \mathrm{~m} \\
\text { tes bearing } \\
\text { foraminifers }\end{array}$} & & \\
\hline & & & $\begin{array}{c}\text { Calcareous turbidites bearing } \\
\text { Santonian/Campanian foraminifers }\end{array}$ & & & \\
\hline & $\begin{array}{c}\text { Globotruncana } \\
\text { fornicata-stuartiformis } \\
\text { Zone }\end{array}$ & Camp. & & & $\begin{array}{l}\text { Below 20-1, } \\
64-66 \mathrm{~cm} \\
176.44 \mathrm{~m}\end{array}$ & \\
\hline & $\begin{array}{c}\text { Zone } \\
\text { (Pessagno) }\end{array}$ & Stage & & & & \\
\hline
\end{tabular}

Figure 4. Summary of biostratigraphy, Sites 576,578 , and 580 .

APPENDIX B

Sample Contents of All Material Examined from Sites 576, 578, 579, 580, and 581

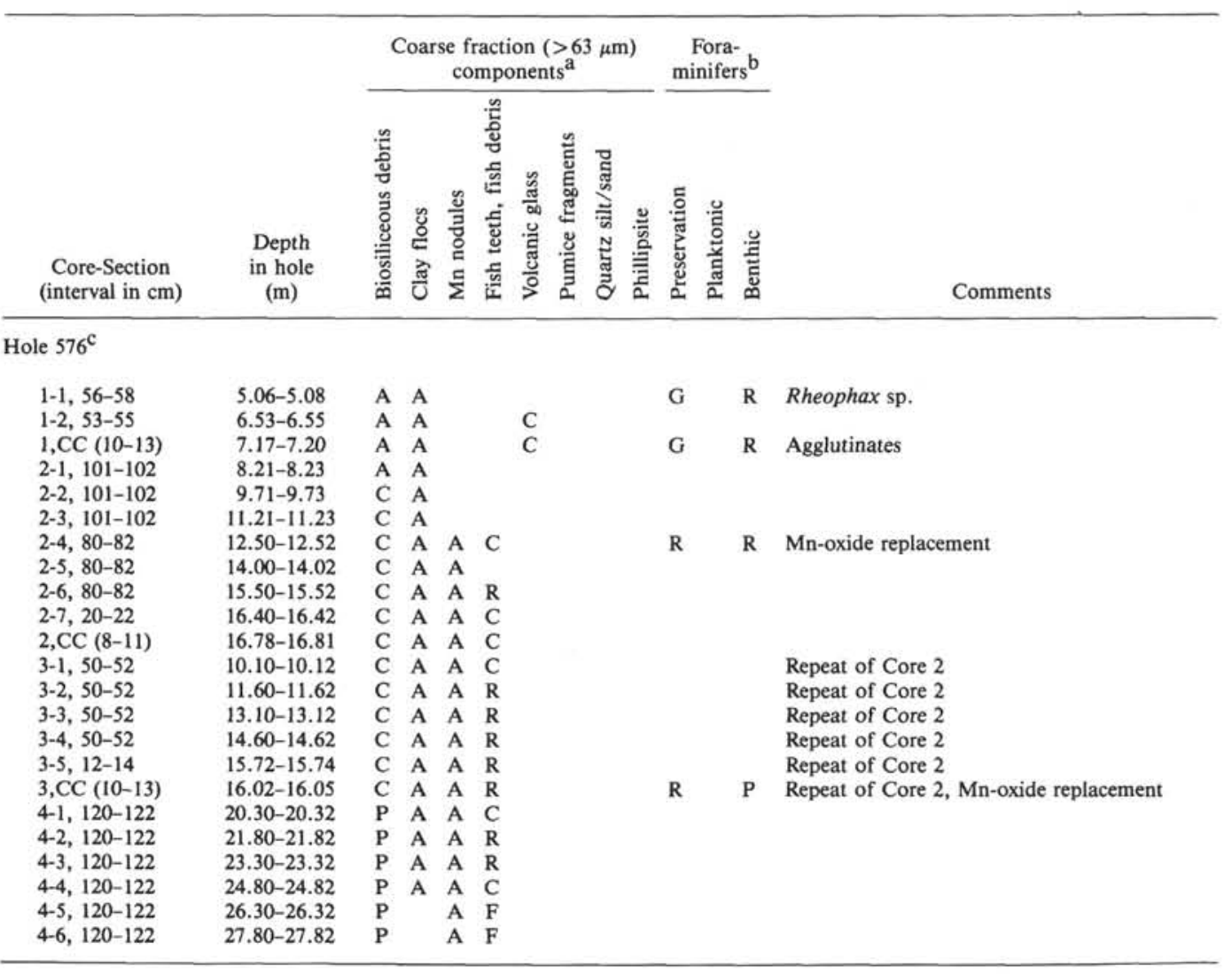




\begin{tabular}{|c|c|c|c|c|c|c|c|c|c|c|c|}
\hline \multirow[b]{2}{*}{$\begin{array}{l}\text { Core-Section } \\
\text { (interval in } \mathrm{cm} \text { ) }\end{array}$} & \multirow[b]{2}{*}{$\begin{array}{l}\text { Depth } \\
\text { in hole } \\
\text { (m) }\end{array}$} & \multicolumn{6}{|c|}{$\begin{array}{l}\text { Coarse fraction }(>63 \mu \mathrm{m}) \\
\text { components }^{\mathrm{a}}\end{array}$} & \multicolumn{3}{|c|}{$\begin{array}{l}\text { Fora- } \\
\text { minifers }\end{array}$} & \multirow[b]{2}{*}{ Comments } \\
\hline & & 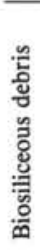 & 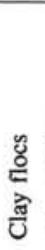 & 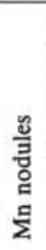 & 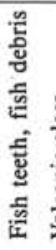 & 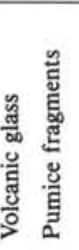 & 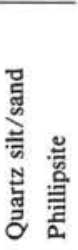 & 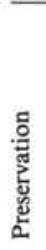 & 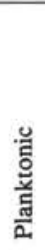 & 总 & \\
\hline \multicolumn{12}{|l|}{ Hole $576^{\mathrm{c}}$ (Cont.) } \\
\hline $4-7,20-22$ & $28.30-28.32$ & $\mathbf{P}$ & & A & & & & & & & \\
\hline $4, C C(10-13)$ & $28.68-28.71$ & P & & A & A & & & & & & \\
\hline $5-1,120-122$ & $29.40-29.42$ & $\mathrm{R}$ & A & A & A & & & & & & \\
\hline $5-2,120-122$ & & & A & A & A & & & & & & Flow-in \\
\hline $5-3,102-104$ & & & A & A & A & & & & & & Flow-in \\
\hline $5-4,102-104$ & & & A & A & A & & & & & & Flow-in \\
\hline $5-5,102-104$ & $32.12-32.14$ & $\mathbf{P}$ & A & A & A & & & & & & \\
\hline $5-6,102-104$ & $33.62-33.64$ & P & A & A & A & & & & & & \\
\hline $5-7,30-32$ & $34.40-34.42$ & P & A & A & A & & & & & & \\
\hline $5, \mathrm{CC}(10-13)$ & $34.69-34.72$ & $\mathbf{P}$ & $\mathrm{F}$ & A & A & & & & & & \\
\hline $6-1,135-136$ & & P & $\mathbf{R}$ & $\mathrm{F}$ & A & & & & & & Flow-in \\
\hline $6-2,112-113$ & & $\mathbf{P}$ & $\mathrm{R}$ & A & A & & A & & & & Flow-in \\
\hline $6-3,112-113$ & $35.52-35.53$ & P & $\mathrm{R}$ & $\mathrm{F}$ & A & & A & & & & \\
\hline $6-4,112-113$ & $37.02-37.03$ & $\mathbf{P}$ & & & A & & & & & & \\
\hline $6-5,112-113$ & $38.52-38.53$ & & $\mathbf{F}$ & $\mathrm{F}$ & A & & & & & & \\
\hline $6-6,112-113$ & $40.02-40.03$ & $\mathbf{P}$ & & & A & & & & & & \\
\hline $6-7,40-42$ & $40.80-40.82$ & & $\mathrm{P}$ & & A & & & & & & \\
\hline $\begin{array}{l}6, \mathrm{CC}(10-13) \\
7-1,50-52\end{array}$ & $\begin{array}{l}40.95-40.98 \\
45.10-45.12\end{array}$ & & $\begin{array}{l}\mathrm{P} \\
\mathrm{P}\end{array}$ & & $\begin{array}{l}\text { A } \\
\text { A }\end{array}$ & & & & & & \\
\hline $7-2,50-52$ & $\begin{array}{l}43.10-43.12 \\
46.10-46.12\end{array}$ & & $P^{P}$ & & $\begin{array}{l}\text { A } \\
\text { A }\end{array}$ & & A & & & & \\
\hline $7-3,50-52$ & $47.60-47.62$ & & A & A & A & & & & & & \\
\hline $7-4,50-52$ & $49.10-49.12$ & & $\mathrm{~F}$ & $\mathrm{~A}$ & A & & & & & & \\
\hline $7.5,50-52$ & $50.60-50.62$ & & $\mathbf{R}$ & $\mathbf{R}$ & A & A & & & & & \\
\hline $7-6,50-52$ & $52.10-52.12$ & P & $\mathbf{R}$ & $\mathbf{R}$ & A & & & & & & \\
\hline $7-7,40-42$ & $53.50-53.52$ & & $\mathrm{~F}$ & $\mathbf{R}$ & A & & & & & & \\
\hline $7, \mathrm{CC}(15-18)$ & $53.75-53.78$ & & $\mathbf{F}$ & & A & & & & & & \\
\hline $8-1,90-92$ & $55.00-55.02$ & & A & A & A & & A & & & & \\
\hline $\begin{array}{l}8-1,135-137 \\
8-2,4-6\end{array}$ & $\begin{array}{l}55.35-55.37 \\
55.64-55.66\end{array}$ & & $\begin{array}{l}\text { A } \\
\text { A }\end{array}$ & $\begin{array}{l}\text { A } \\
\text { A }\end{array}$ & $\begin{array}{l}\text { A } \\
\text { A }\end{array}$ & & A & M & & $\mathbf{R}$ & \\
\hline $8-2,18-20$ & $55.78-55.80$ & & & A & A & & & M & A & $\mathrm{C}$ & $\begin{array}{l}\text { Graded nannofossil ooze, tests in } 63-149 \mu \mathrm{m} \\
\quad \text { fraction, dissolution, abrasion }\end{array}$ \\
\hline $8-2,25-27$ & $55.85-55.87$ & & A & A & & & & M & C & $\mathrm{C}$ & Bioturbated clay \\
\hline $8-2,50-52$ & $56.10-56.12$ & & $\mathbf{R}$ & A & A & & & M & A & $\mathrm{F}$ & Dissolution, graded nannofossil ooze \\
\hline $8-2,71-73$ & $56.31-56.33$ & & & A & A & & A & G & $\mathbf{R}$ & $\mathrm{C}$ & Tests stained brown, bioturbated clay \\
\hline $8-2,88-90$ & $56.48-56.50$ & & $\mathbf{R}$ & A & A & & & $\mathrm{M}$ & A & C & Abrasion, dissolution, nannofossil ooze \\
\hline $8-2,132-134$ & $56.92-56.94$ & & & $\mathrm{C}$ & A & & & M & $\mathbf{R}$ & $\mathrm{C}$ & Brown tests, abrasion, bioturbated clay \\
\hline $8-3,18-20$ & $57.28-57.30$ & & $\mathbf{R}$ & C & A & & & M & $\mathbf{R}$ & $\mathrm{F}$ & Bioturbated nannofossil ooze \\
\hline $8-3,66-68$ & $57.76-57.78$ & & & $\mathrm{C}$ & C & & A & & & & \\
\hline $8-3,81-83$ & $57.91-57.93$ & & $\mathbf{R}$ & $\mathrm{F}$ & & & A & & & & \\
\hline $8-3,96-98$ & $58.06-68.08$ & & & $\mathrm{~F}$ & A & & & & & & \\
\hline $8-3,122-124$ & $58.32-58.34$ & & & $\mathbf{F}$ & C & & & M & C & C & Nannofossil ooze \\
\hline $8-4,10-12$ & $58.78-58.72$ & & & & & & & $\mathrm{M}$ & $\mathrm{R}$ & $\mathrm{C}$ & Brown-stain, nannofossil ooze, bioturbation? \\
\hline $8-4,20-22$ & $58.80-58.82$ & & & A & A & & & & & & \\
\hline $8-4,30-32$ & $58.90-58.92$ & $\mathbf{P}$ & & & & & & $\mathbf{P}$ & A & $\mathrm{F}$ & Nannofossil ooze, dissolution, abrasion \\
\hline $8-4,49-51$ & $59.09-59.11$ & P & & $\mathbf{R}$ & & & & $\mathbf{P}$ & A & $\mathrm{C}$ & Nannofossil ooze, dissolution, abrasion \\
\hline $8-4,110-112$ & $59.70-59.72$ & P & & $\mathbf{R}$ & & & & $\mathbf{P}$ & A & C & Nannofossil ooze, dissolution, abrasion \\
\hline $8-5,12-14$ & $60.22-60.24$ & & & $\mathbf{R}$ & & & & P & A & C & Nannofossil ooze, dissolution, abrasion \\
\hline $8-5,132-134$ & $61.42-61.44$ & $\mathbf{P}$ & & $\mathrm{C}$ & & & & $\mathbf{P}$ & A & C & Nannofossil ooze, dissolution, abrasion \\
\hline $8-6,12-14$ & $61.72-61.74$ & P & & $\mathrm{C}$ & & & & $\mathbf{P}$ & A & C & Nannofossil ooze, dissolution, abrasion \\
\hline $8-6,42-44$ & $62.02-62.04$ & & & $\mathrm{C}$ & & & & $\mathbf{P}$ & A & C & Nannofossil ooze, dissolution, abrasion \\
\hline $8-6,52-54$ & $62.12-62.14$ & $\mathbf{R}$ & & & & & & $\mathbf{P}$ & A & $\mathrm{C}$ & Cross-bedding, dissolution, abrasion \\
\hline $8-6,62-64$ & $62.22-62.24$ & $\mathbf{R}$ & & & & & & $\mathbf{P}$ & A & $\mathrm{C}$ & Graded nannofossil ooze \\
\hline $8-6,72-74$ & $62.32-62.34$ & $\mathbf{R}$ & & $\mathrm{C}$ & A & & & $\mathbf{P}$ & A & $\mathrm{C}$ & Graded nannofossil ooze, bioturbated? \\
\hline $8-6,84-86$ & $62.44-62.46$ & $\mathbf{R}$ & & C & A & & & $\mathbf{P}$ & A & $\mathrm{C}$ & Graded nannofossil ooze, bioturbated? \\
\hline $8-6,98-100$ & $62.58-62.60$ & $\mathbf{P}$ & $\mathbf{F}$ & C & $\mathbf{R}$ & & & P & A & C & $\begin{array}{l}\text { White tests, dissolution, abrasion, bioturbated } \\
\text { nannofossil ooze }\end{array}$ \\
\hline $8-6,125-127$ & $62.85-62.87$ & $\mathbf{P}$ & $\mathbf{F}$ & & A & & & $\mathbf{P}$ & A & C & $\begin{array}{l}\text { White tests, dissolution, abrasion, bioturbated } \\
\text { clay }\end{array}$ \\
\hline $8-6,148-150$ & $62.08-63.10$ & $\mathbf{P}$ & & $\mathrm{F}$ & & & & $\mathbf{P}$ & A & C & $\begin{array}{l}\text { White tests, dissolution, abrasion, bioturbated } \\
\text { nannofossil ooze }\end{array}$ \\
\hline $8-7,16-18$ & $63.26-63.28$ & $\mathbf{P}$ & & C & & & & $\mathbf{P}$ & A & $\mathrm{C}$ & $\begin{array}{l}\text { White tests, dissolution, abrasion, bioturbated } \\
\text { nannofossil ooze }\end{array}$ \\
\hline $8, \mathrm{CC}(4-7)$ & $63.57-63.59$ & $\mathbf{R}$ & $\mathrm{C}$ & C & A & & A & $\mathbf{P}$ & $\mathbf{F}$ & $\mathrm{F}$ & Broken tests stained brown \\
\hline $8, \mathrm{CC}(15-18)$ & $63.68-63.70$ & & & $\mathbf{F}$ & & & & $\mathrm{P}$ & A & C & $\begin{array}{l}\text { White tests, dissolution, abrasion, nannofossil } \\
\text { ooze }\end{array}$ \\
\hline Foram-bearing & & & & & & & & & & & \\
\hline
\end{tabular}


Appendix B. (Continued).

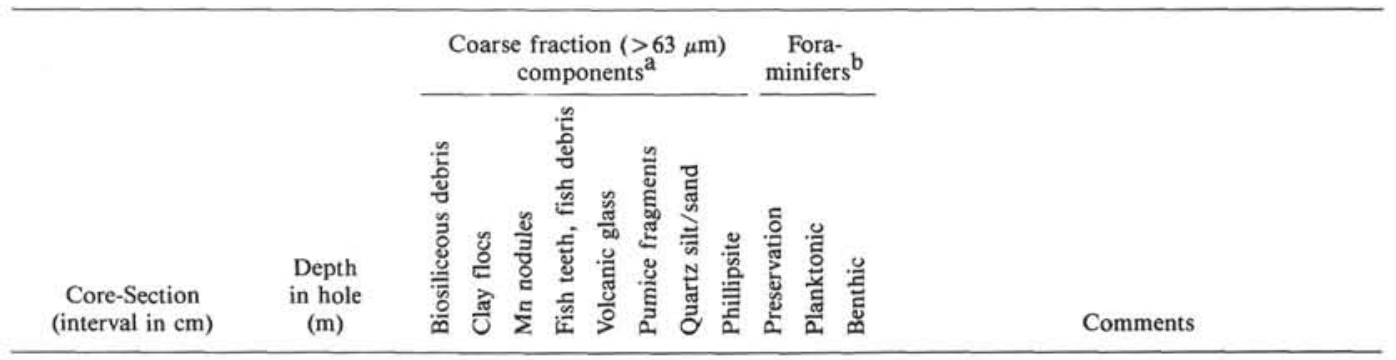

Hole $576^{\mathrm{c}}$

Shipboard CC's

$8, \mathrm{CC}$

Hole $576 \mathrm{~B}^{\mathrm{c}}$

\begin{tabular}{|c|c|c|c|c|c|c|}
\hline $1-1,108-110$ & $1.08-1.10$ & A & A & & & \\
\hline $1-2,108-110$ & $2.58-2.60$ & A & A & & & $\mathbf{R}$ \\
\hline $1-3,108-110$ & $4.08-4.10$ & A & A & F & & \\
\hline $1-4,108-110$ & $5.58-5.60$ & A & A & F & & \\
\hline $1-5,68-70$ & $6.68-6.70$ & A & A & $\mathrm{F}$ & $\mathbf{P}$ & \\
\hline $1-6,20-22$ & $7.70-7.72$ & A & A & F & & \\
\hline $1, \mathrm{CC}(7-10)$ & $8.14-8.16$ & A & A & A & & \\
\hline $2-1,130-132$ & $12.90-12.92$ & A & A & A & $\mathbf{R}$ & \\
\hline $2-2,130-132$ & $14.40-14.42$ & A & A & A & $\mathbf{R}$ & \\
\hline $2-3,130-132$ & $15.90-15.92$ & A & A & A & $\mathbf{R}$ & \\
\hline $2-4,130-132$ & $17.40-17.42$ & A & A & A & $\mathbf{R}$ & \\
\hline $2-5,130-132$ & $18.90-18.92$ & R & A & A & $R$ & \\
\hline $2-6,130-132$ & $20.40-20.42$ & $\mathbf{R}$ & A & A & $\mathbf{R}$ & \\
\hline $2-7,10-12$ & $20.70-20.72$ & & A & A & $\mathbf{R}$ & \\
\hline $2, \mathrm{CC}(10-13)$ & $21.08-21.10$ & & A & A & $\mathrm{F}$ & \\
\hline $3-1,92-94$ & $22.02-22.04$ & $\mathrm{P}$ & A & A & $\mathbf{R}$ & \\
\hline $3-2,92-94$ & $23.52-23.54$ & & A & A & $\mathbf{R}$ & \\
\hline $3-3,92-94$ & $25.02-25.04$ & & P & A & & \\
\hline $3-4,92-94$ & & & A & A & $\mathbf{R}$ & \\
\hline $3-5,92-94$ & & & & A & A & \\
\hline $3-6,92-94$ & $25.41-25.43$ & & A & A & A & \\
\hline $3-7,10-12$ & $26.09-26.11$ & & C & A & A & \\
\hline $3, \mathrm{CC}(10-13)$ & $26.59-26.61$ & & C & A & A & \\
\hline $4-1,138-140$ & & & A & A & A & \\
\hline $4-2,12-14$ & & & A & A & A & \\
\hline $4-3,12-14$ & & & A & A & A & \\
\hline $4-4,12-14$ & $34.72-34.74$ & & A & A & A & \\
\hline $4-5,12-14$ & $36.22-36.24$ & & & A & A & \\
\hline $4-6,12-14$ & $37.72-37.74$ & & & A & A & \\
\hline $4-7,12-14$ & $39.22-39.24$ & & & A & A & \\
\hline $4, \mathrm{CC}(10-13)$ & $39.72-39.74$ & & & C & A & \\
\hline $5-1,130-132$ & & & $\mathbf{R}$ & & A & \\
\hline $5-2,34-36$ & $40.44-40.46$ & & $\mathrm{R}$ & $\mathrm{R}$ & A & \\
\hline $5-3,34-36$ & $41.94-41.96$ & & $\mathbf{R}$ & $\mathrm{R}$ & A & \\
\hline $5-4,34-36$ & $43.44-43.46$ & & $\mathbf{R}$ & R & A & \\
\hline $5-5,34-36$ & $44.94-44.96$ & & $\mathbf{R}$ & & A & \\
\hline $5-6,34-36$ & $46.44-46.46$ & & $\mathrm{R}$ & $\mathrm{R}$ & A & \\
\hline $5, \mathrm{CC}(10-13)$ & $47.43-47.45$ & & $\mathbf{R}$ & A & A & \\
\hline $6-1,110-112$ & & & $P$ & C & C & \\
\hline $6-2,110-112$ & $52.20-52.22$ & & $\mathbf{R}$ & A & A & \\
\hline $6-3,110-112$ & $53.70-53.72$ & & & $\mathrm{R}$ & A & \\
\hline $6-4,90-92$ & $55.00-55.02$ & & & A & A & \\
\hline $6-4,110-112$ & $55.20-55.22$ & & A & A & A & \\
\hline $6-4,126-128$ & $55.36-55.38$ & & C & C & C & \\
\hline $6-5,24-26$ & $55.84-55.86$ & & C & C & & \\
\hline $6-5,56-58$ & $56.16-56.18$ & & & C & $\mathbf{R}$ & \\
\hline $6-5,70-72$ & $56.30-56.32$ & & & C & $\mathrm{R}$ & \\
\hline $6-5,96-98$ & $56.56-56.58$ & & & A & A & \\
\hline $6-6,28-30$ & $57.38-57.40$ & & & $\mathrm{C}$ & C & \\
\hline $6-6,58-60$ & $57.68-57.70$ & & & $\mathbf{R}$ & $\mathbf{R}$ & R \\
\hline $6-6,100-102$ & $58.10-58.12$ & & & $\mathbf{R}$ & $\mathbf{R}$ & \\
\hline $6, \mathrm{CC}(34-35)$ & $58.86-58.88$ & & & $\mathbf{P}$ & $\mathbf{R}$ & \\
\hline $7-1,76-78$ & $59.84-59.86$ & & $\mathrm{R}$ & $\mathbf{R}$ & & \\
\hline $7-2,76-78$ & $61.34-61.36$ & $P$ & $\mathbf{R}$ & $\mathbf{R}$ & $\mathrm{R}$ & \\
\hline
\end{tabular}

M A R
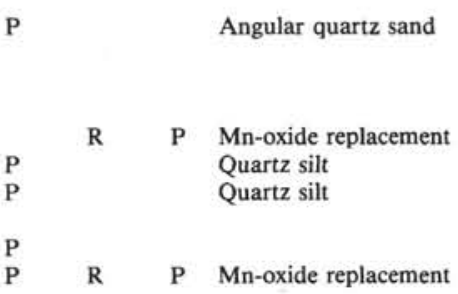

Flow-in

Flow-in

Flow-in

Flow-in

Flow-in

Flow-in

Flow-in

A Quartz silt

M F F Dissolution, abrasion, white tests, nannofossil ooze

P C F Dissolution, abrasion, white tests, bioturbated

P C F Dissolution, abrasion, white tests, bioturbated nannofossil ooze

P R Tests stained brown, bioturbated clay

$\begin{array}{llll}M & R & R\end{array}$

P $\quad$ R R Dissolution, abrasion, white tests, bioturbated nannofossil ooze

P A F Dissolution, abrasion, nannofossil ooze, white tests

P A C Dissolution, abrasion, nannofossil ooze, white tests

P A C Dissolution, abrasion, nannofossil ooze, white tests 


\begin{tabular}{|c|c|c|c|c|c|c|c|c|c|c|c|c|}
\hline \multirow[b]{2}{*}{$\begin{array}{l}\text { Core-Section } \\
\text { (interval in } \mathrm{cm} \text { ) }\end{array}$} & \multirow[b]{2}{*}{$\begin{array}{l}\text { Depth } \\
\text { in hole } \\
\text { (m) }\end{array}$} & \multicolumn{7}{|c|}{$\begin{array}{l}\text { Coarse fraction }(>63 \mu \mathrm{m}) \\
\text { components }^{\mathrm{a}}\end{array}$} & \multicolumn{3}{|c|}{$\begin{array}{c}\text { Fora- } \\
\text { minifers }\end{array}$} & \multirow[b]{2}{*}{ Comments } \\
\hline & & 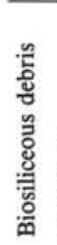 & 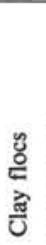 & है & 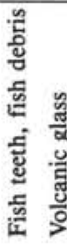 & 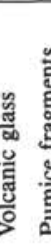 & 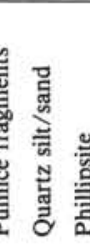 & 葪 & 吾。 & 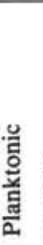 & 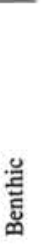 & \\
\hline \multicolumn{13}{|l|}{ Hole $576 \mathrm{~B}^{\mathrm{c}}$ (Cont.) } \\
\hline $7-3,115-117$ & $62.15-62.17$ & $\mathrm{P}$ & $\mathbf{R}$ & $\mathrm{R}$ & $\mathrm{P}$ & & & & $\mathbf{P}$ & A & $\mathrm{C}$ & $\begin{array}{l}\text { Dissolution, abrasion, nannofossil ooze, white } \\
\text { tests }\end{array}$ \\
\hline $7-4,24-26$ & $62.74-62.76$ & $\mathrm{C}$ & $\mathbf{R}$ & & & & & & P & A & $\mathrm{C}$ & $\begin{array}{l}\text { Dissolution, abrasion, nannofossil ooze, white } \\
\text { tests }\end{array}$ \\
\hline $7-4,115-117$ & $63.65-63.67$ & $\mathbf{R}$ & & $\mathbf{R}$ & R & & & & P & A & C & $\begin{array}{l}\text { Dissolution, abrasion, nannofossil ooze, white } \\
\text { tests }\end{array}$ \\
\hline $7-5,104-106$ & $65.04-65.06$ & $\mathrm{R}$ & $\mathbf{R}$ & & & & & & $\mathbf{P}$ & A & $\mathrm{C}$ & $\begin{array}{l}\text { Dissolution, abraison, bioturbated nannofos- } \\
\text { sil ooze, white tests }\end{array}$ \\
\hline $7-6,68-70$ & $66.18-66.20$ & & $\mathbf{R}$ & $\mathrm{R}$ & & & & & $\mathbf{P}$ & $\mathrm{R}$ & $\mathbf{R}$ & Fragments only \\
\hline $7-6,120-122$ & $66.70-66.72$ & $\mathrm{P}$ & 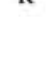 & $\mathrm{R}$ & & & & & $\mathrm{P}$ & $\mathrm{F}$ & $\mathrm{F}$ & $\begin{array}{l}\text { Dissolution, abrasion, nannofossil ooze, white } \\
\text { tests }\end{array}$ \\
\hline $7-7,10-12$ & $67.10-67.12$ & $\mathbf{P}$ & & $\mathrm{R}$ & & & & & P & A & $\mathrm{C}$ & $\begin{array}{l}\text { Dissolution, abrasion, nannofossil ooze, white } \\
\text { tests }\end{array}$ \\
\hline $7, \mathrm{CC}(30-33)$ & $67.64-67.66$ & $\mathrm{P}$ & $\mathbf{R}$ & $\mathbf{R}$ & & & & & $\mathbf{P}$ & A & $\mathrm{C}$ & $\begin{array}{l}\text { Dissolution, abrasion, nannofossil ooze, white } \\
\text { tests }\end{array}$ \\
\hline $8-1,135-140$ & $68.80-68.82$ & $\mathrm{P}$ & & $\mathrm{P}$ & & & & & $\mathbf{P}$ & A & C & $\begin{array}{l}\text { Dissolution, abrasion, nannofossil ooze, white } \\
\text { tests }\end{array}$ \\
\hline $8-2,58-60$ & $69.53-69.55$ & $\mathrm{P}$ & $\mathbf{R}$ & $\mathrm{R}$ & & & & & $\mathbf{P}$ & A & $\mathrm{C}$ & $\begin{array}{l}\text { Dissolution, abrasion, nannofossil ooze, white } \\
\text { tests }\end{array}$ \\
\hline $8-2,128-130$ & $70.23-70.25$ & & & & & & & & $\mathbf{P}$ & $\mathbf{R}$ & $\mathbf{R}$ & $\begin{array}{l}\text { Dissolution, abrasion, } 63-149 \mu \mathrm{m} \text { fraction } \\
\text { only, graded nannofossil ooze }\end{array}$ \\
\hline $8-3,50-52$ & $70.95-70.97$ & & & & & & & & $P$ & $\mathrm{~F}$ & $\mathbf{R}$ & $\begin{array}{l}\text { Dissolution, abrasion, 63-149 } \mu \mathrm{m} \text { fraction } \\
\text { only }\end{array}$ \\
\hline $8-3,124-126$ & $71.69-71.71$ & & $\mathbf{R}$ & & $\mathrm{R}$ & & & & $\mathbf{P}$ & $\mathrm{F}$ & $\mathbf{R}$ & $\begin{array}{l}\text { Dissolution, abrasion, } 63-149 \mu \mathrm{m} \text { fraction } \\
\text { only }\end{array}$ \\
\hline $8-4,66-68$ & $72.61-72.63$ & & & & & & & & P & $\mathbf{F}$ & $\mathbf{R}$ & $\begin{array}{l}\text { Dissolution, abrasion, } 63-149 \mu \mathrm{m} \text { fraction } \\
\text { only }\end{array}$ \\
\hline $8-4,124-126$ & $73.19-73.21$ & & R & & $\mathrm{P}$ & & & & P & $\mathrm{F}$ & $\mathbf{R}$ & $\begin{array}{l}\text { Dissolution, abrasion, } 63-149 \mu \mathrm{m} \text { fraction } \\
\text { only, graded ooze }\end{array}$ \\
\hline $8-5,90-92$ & $74.35-74.37$ & & $\mathrm{R}$ & & & & & & P & & $\mathbf{R}$ & $\begin{array}{l}\text { Tests stained brown, dissolution, bioturba- } \\
\text { tion? }\end{array}$ \\
\hline \multicolumn{13}{|r|}{ tront } \\
\hline $7, \mathrm{CC}$ & & & & & & & & & $\mathbf{P}$ & & $\mathbf{R}$ & Agglut. benthics \\
\hline $6, \mathrm{CC}$ & & & & & & & & & M & & $\mathbf{R}$ & Agglut. benthics \\
\hline \multicolumn{13}{|l|}{ Hole 578} \\
\hline $1-3,80-82$ & $3.80-3.82$ & A & A & & & & & & G & & $\mathbf{R}$ & 2 calc. benthic, 1 agglut. \\
\hline $1, \mathrm{CC}(18-20)$ & $4.78-4.80$ & $\mathrm{C}$ & A & & $\mathbf{R}$ & & & & & & & \\
\hline $2-3,80-82$ & $8.60-8.62$ & A & A & & & & & & & & & \\
\hline $2, \mathrm{CC}(18-20)$ & $14.28-14.30$ & A & A & & & A & & & & & & \\
\hline $3-3,90-92$ & $18.20-18.22$ & A & A & & & & & & & & & \\
\hline $3, \mathrm{CC}(18-20)$ & $23.70-23.72$ & A & A & $\mathbf{R}$ & & & & & & & & \\
\hline $4-3,90-92$ & $27.70-27.72$ & A & A & & $\mathbf{R}$ & & & & & & & \\
\hline $4, \mathrm{CC}(18-20)$ & $33.28-33.30$ & A & A & & & & & & & & & \\
\hline $5-4,90-92$ & $38.70-38.72$ & A & A & & & & & & & & & \\
\hline $5, \mathrm{CC}(18-20)$ & $42.78-42.80$ & A & A & & & A & & & & & & \\
\hline $6-4,70-72$ & $48.00-48.02$ & A & A & & & A & & & & & & \\
\hline $6, \mathrm{CC}(18-20)$ & $52.58-52.30$ & A & A & & & A & & & & & & \\
\hline $7-2,70-72$ & $54.50-54.52$ & A & A & & & A & & & & & & \\
\hline $7, \mathrm{CC}(18-20)$ & $61.78-61.80$ & A & A & & & & & & & & & \\
\hline $8-3,60-62$ & $65.40-65.42$ & A & A & & & $\mathrm{C}$ & & & & & & \\
\hline $8, \mathrm{CC}(18-20)$ & $71.28-71.30$ & A & A & & & A & & & & & & $\begin{array}{l}\text { Green and clear glassy particles }>149 \mu \mathrm{m} \text { in } \\
\text { size }\end{array}$ \\
\hline $9-3,57-59$ & $74.87-74.89$ & A & A & & & A & & & & & & \\
\hline $9, \mathrm{CC}(18-20)$ & $80.78-80.80$ & A & A & & & A & & A & & & & \\
\hline $10-3,69-71$ & $84.49-84.51$ & $\mathrm{~A}$ & A & $\mathrm{C}$ & & A & & & & & & \\
\hline $10, \mathrm{CC}(18-20)$ & $90.28-90.30$ & A & A & $\mathrm{C}$ & & A & $P$ & & & & & \\
\hline $11-3,60-62$ & $93.90-93.92$ & $\mathrm{~A}$ & A & & & & & A & & & & \\
\hline $11, \mathrm{CC}(18-20)$ & $99.78-99.90$ & A & A & & & & & & & & & \\
\hline $12-3,50-52$ & $103.30-103.32$ & A & A & & & & & & & & & \\
\hline $12, \mathrm{CC}(18-20)$ & $109.28-109.30$ & A & A & A & & & & A & G & & $\mathbf{R}$ & 2 calc. benthic \\
\hline $13-4,80-82$ & $114.60-114.62$ & A & & & & A & & & & & & \\
\hline $13, \mathrm{CC}(18-20)$ & $118.78-118.80$ & A & A & A & $\mathrm{R}$ & & & A & & & & \\
\hline $14-3,90-92$ & $122.70-122.72$ & A & A & $\mathrm{C}$ & R & & & A & & & & \\
\hline $14, \mathrm{CC}(18-20)$ & $128.28-128.30$ & & & A & $\mathrm{R}$ & & & & & & & \\
\hline
\end{tabular}


Appendix B. (Continued).

\begin{tabular}{|c|c|c|c|c|c|c|}
\hline \multirow[b]{2}{*}{$\begin{array}{c}\text { Core-Section } \\
\text { (interval in } \mathrm{cm} \text { ) }\end{array}$} & \multirow[b]{2}{*}{$\begin{array}{c}\text { Depth } \\
\text { in hole } \\
\text { (m) }\end{array}$} & \multicolumn{3}{|c|}{$\begin{array}{l}\text { Coarse fraction }(>63 \mu \mathrm{m}) \\
\text { components }^{\mathrm{a}}\end{array}$} & $\begin{array}{c}\text { Fora- } \\
\text { minifers }\end{array}$ & \\
\hline & & 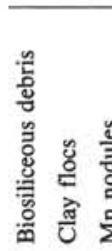 & 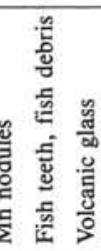 & 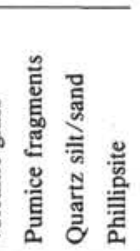 & 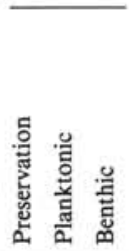 & Comments \\
\hline
\end{tabular}

Hole 578 (Cont.)

$\begin{array}{ll}15-3,33-35 & 131.63-131.65 \\ 15, C C(18-20) & 137.78-137.80 \\ 16-1,20-22 & 138.00-138.02 \\ 16-2,28-30 & 139.58-139.60 \\ 16-2,138-139 & 140.68-140.69 \\ 16-3,130-131 & 142.09-142.11 \\ 16-5,138-139 & 145.17-145.19 \\ 16, C C(18-20) & 147.28-147.30 \\ 17, C C(18-20) & 155.28-155.30 \\ 18-3,50-52 & 160.30-160.32 \\ 18, C C(18-20) & 163.28-163.30 \\ 19-2,70-72 & 168.50-168.52 \\ 20-1,64-66 & 176.44-176.46 \\ 20, C C & 176.78-176.80\end{array}$

A A A R

A A $R$

A A R

A A $R$

A A R

A A R

A A A C

R A A

C A

A A

A A
Blocky, varicolored glass

M C R late early to early middle Eocene

R A C Silica replacement, Campanian

R A C Silica replacement, Campanian
Hole 579

$\begin{array}{lcccc}1-4,41-43 & 4.91-4.93 & & \text { A } & \text { P } \\ 1, C C(18-20) & 8.38-8.40 & & \text { A } & \text { A } \\ 2-3,95-97 & 12.35-12.37 & & \text { A } & \text { P } \\ 2, C C ~(18-20) & 17.88-17.99 & \text { A } & \text { A } & \text { R }\end{array}$

Hole 579A

\begin{tabular}{|c|c|c|}
\hline 5. & 20.97 & A \\
\hline $1, C C(18-20)$ & $23.48-23.50$ & A \\
\hline $2-5,100-102$ & $30.50-30.52$ & A \\
\hline $2, \mathrm{CC}(18-20)$ & $32.98-33.00$ & A \\
\hline $3-3,68-70$ & $36.68-36.70$ & A \\
\hline $3, C C(18-20)$ & $42.48-42.50$ & C \\
\hline $4-3,118-120$ & $46.68-46.70$ & A \\
\hline $4, \mathrm{CC}(18-20)$ & $51.98-51.20$ & A \\
\hline $5-3,53-55$ & $55.53-55.55$ & A \\
\hline $5, \mathrm{CC}(18-20)$ & $61.48-61.50$ & A \\
\hline $6-3,50-52$ & $65.00-65.02$ & A \\
\hline $6, \mathrm{CC}(18-20)$ & $70.98-71.00$ & A \\
\hline $7-3,94-96$ & $74.94-74.96$ & A \\
\hline $7, \mathrm{CC}(18-20)$ & $80.48-80.50$ & A \\
\hline $8-5,100-102$ & $87.50-87.52$ & A \\
\hline $8, \mathrm{CC}(18-20)$ & $89.98-90.00$ & A \\
\hline $9-3,70-72$ & $93.70-93.72$ & A \\
\hline $9, \mathrm{CC}(18-20)$ & $99.48-99.50$ & A \\
\hline $10-4,100-102$ & $105.00-105.02$ & A \\
\hline $10, \mathrm{CC}(18-20)$ & $108.98-109.00$ & $\mathrm{C}$ \\
\hline $11, C C(18-20)$ & $118.48-118.50$ & A \\
\hline $12-3,28-30$ & $121.78-121.80$ & A \\
\hline $12, \mathrm{CC}(18-20)$ & $127.98-128.00$ & A \\
\hline $13-3,70-72$ & $131.70-131.72$ & \\
\hline $13, \mathrm{CC}(18-20)$ & $137.48-137.50$ & A \\
\hline $14-3,30-32$ & $140.80-140.82$ & \\
\hline $14, \mathrm{CC}(18-20)$ & $146.98-147.00$ & A \\
\hline $15-1,110-112$ & $148.10-148.12$ & A \\
\hline $15, \mathrm{CC}(18-20)$ & $149.48-149.50$ & A \\
\hline
\end{tabular}

A A

A

A

A $\mathrm{A}$

A A C

A

A

A A

A A

A A

A

A

$A$

A A

A A

A
A
C

Hole 580

\begin{tabular}{|c|c|c|c|c|c|c|}
\hline $1-1,27-29$ & $0.27-0.29$ & A & $\mathrm{C}$ & & & \\
\hline $1-1,112-114$ & $1.12-1.14$ & A & A $\mathrm{C}$ & G & $\mathbf{P}$ & 2 calc. benthics, 1 rounded basalt pebble \\
\hline $1-2,33-35$ & $1.83-1.85$ & A & A & G A & A & Rounded basalt pebbles \\
\hline $1-2,142-144$ & $2.92-2.94$ & A A & $\mathrm{C}$ & & & \\
\hline $1-3,3-5$ & $3.03-3.05$ & A & $\mathrm{C}$ & & & 2 rounded basalt pebbles \\
\hline $1, \mathrm{CC}(18-20)$ & $3.28-3.30$ & A & $\mathrm{C}$ & & & \\
\hline $2-1,76-80$ & $4.06-4.08$ & A & A & & & \\
\hline $2-2,124-126$ & $6.04-6.06$ & A & A & & & \\
\hline $2-3,108-110$ & $7.38-7.40$ & A & $\mathrm{C}$ & & & \\
\hline $2-3,124-126$ & $7.54-7.56$ & A & $\mathrm{C}$ & & & \\
\hline
\end{tabular}


Appendix B. (Continued).

\begin{tabular}{|c|c|c|c|c|c|c|c|c|c|c|c|}
\hline \multirow[b]{2}{*}{$\begin{array}{l}\text { Core-Section } \\
\text { (interval in } \mathrm{cm} \text { ) }\end{array}$} & \multirow[b]{2}{*}{$\begin{array}{l}\text { Depth } \\
\text { in hole } \\
\text { (m) }\end{array}$} & \multicolumn{6}{|c|}{$\begin{array}{c}\text { Coarse fraction }(>63 \mu \mathrm{m}) \\
\text { components }^{\mathrm{a}}\end{array}$} & \multicolumn{3}{|c|}{$\begin{array}{c}\text { Fora- } \\
\text { minifers }\end{array}$} & \multirow[b]{2}{*}{ Comments } \\
\hline & & 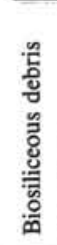 & 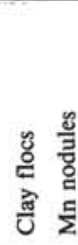 & 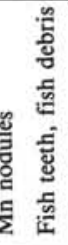 & 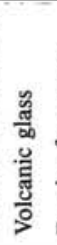 & 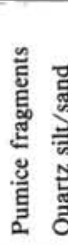 & 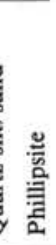 & 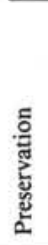 & $\begin{array}{l}\stackrel{\mathscr{Z}}{\tilde{\mathscr{Z}}} \\
\frac{\ddot{m}}{\mathscr{a}}\end{array}$ & 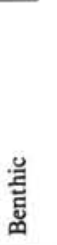 & \\
\hline \multicolumn{12}{|l|}{ Hole 580 (Cont.) } \\
\hline $2-4,34-36$ & $8.14-8.16$ & A & A & & $\mathrm{C}$ & & & & & & \\
\hline $2-4,106-108$ & $8.86-8.88$ & A & & & A & & & G & A & $\mathrm{C}$ & \\
\hline $2-5,72-74$ & $10.02-10.04$ & $\mathrm{~A}$ & & & A & & & & & & \\
\hline $2, \mathrm{CC}(18-20)$ & $12.78-12.80$ & $\mathrm{~A}$ & & & A & & & & & & \\
\hline $3-4,45-47$ & $17.75-17.77$ & $\mathrm{~A}$ & A & & & & & & & & \\
\hline $3, C C(18-20)$ & $22.28-22.30$ & A & $\mathrm{C}$ & & $\mathrm{C}$ & & & & & & Varicolored glassy grains \\
\hline $4-3,80-82$ & $26.10-26.12$ & A & A & & & & & G & A & $\mathbf{R}$ & \\
\hline $4, C C(18-20)$ & $31.78-31.80$ & A & & & A & & & & & & \\
\hline $5-5,85-87$ & $38.65-38.67$ & A & A & & & & & G & $\mathbf{R}$ & $\mathbf{R}$ & \\
\hline $5, C C(18-20)$ & $41.28-41.30$ & A & A & & & & & $\mathrm{M}$ & $\mathbf{R}$ & $\mathbf{R}$ & \\
\hline $6-3,58-60$ & $44.88-44.90$ & A & A & & & & & M & A & C & \\
\hline $6, C C(18-20)$ & $50.78-50.80$ & A & A & & & & & & & & \\
\hline $7-3,62-64$ & $54.42-54.44$ & A & A & & A & & & & & & \\
\hline $7, C C(18-20)$ & $60.28-60.30$ & A & A & & & & & & & & \\
\hline $8-3,43-45$ & $63.73-63.75$ & A & A & & & & & & & & \\
\hline $8, \mathrm{CC}(18-20)$ & $69.78-69.80$ & A & & & A & & & & & & \\
\hline $9-3,32-34$ & $73.12-73.14$ & A & A & & A & & & & & & \\
\hline $9, C C(18-20)$ & $79.28-79.30$ & $\mathrm{~A}$ & & & & & & & & & \\
\hline $10-3,60-62$ & $82.90-82.92$ & A & & & A & & & & & & \\
\hline $10, \mathrm{CC}(18-20)$ & $88.78-88.80$ & A & A & & A & & & & & & \\
\hline $11-3,105-107$ & $92.85-92.87$ & A & A & & & & & & & & \\
\hline $11, \mathrm{CC}(18-20)$ & $98.28-98.30$ & A & A & & A & & & & & & \\
\hline $12-4,20-22$ & $103.00-103.02$ & A & A & & & & & & & & \\
\hline $12, \mathrm{CC}(18-20)$ & $107.78-107.80$ & A & & & & & & & & & \\
\hline $13-4,60-62$ & $112.90-112.92$ & A & & & & & & & & & \\
\hline $13, \mathrm{CC}(18-20)$ & $117.28-117.30$ & A & & & & & & & & & \\
\hline $14-3,30-32$ & $120.60-120.62$ & A & A & & & & & & & & \\
\hline $14, C C(18-20)$ & $126.78-126.80$ & A & C & & & & & & & & \\
\hline $15-2,101-103$ & $129.31-129.33$ & A & & & & & & & & & \\
\hline $15, \mathrm{CC}(18-20)$ & $136.28-136.30$ & A & & & A & & & & & & \\
\hline $16-3,26-28$ & $139.56-139.58$ & A & A & & & & & & & & \\
\hline $16, \mathrm{CC}(18-20)$ & $145.78-145.80$ & A & A & & & & & & & & \\
\hline $17-3,21-23$ & $149.01-149.03$ & A & A & & & & & & & & \\
\hline $17, \mathrm{CC}(18-20)$ & $155.28-155.30$ & A & & & & & & & & & \\
\hline \multicolumn{12}{|l|}{ Hole 581} \\
\hline $1-1,40-42$ & $0.40-0.42$ & A & $\mathrm{C}$ & & A & $\mathrm{C}$ & & & & & Glassy grains of various colors \\
\hline $1, C C(18-20)$ & $0.98-1.00$ & A & $\mathrm{C}$ & & A & $\mathrm{C}$ & & & & & Glassy grains of various colors \\
\hline $2-2,20-22$ & $183.20-183.22$ & A & & & & & & & & & \\
\hline $2, \mathrm{CC}(18-20)$ & $190.98-191.00$ & $\mathrm{~A}$ & $\mathrm{C}$ & & & & & & & & \\
\hline $3-4,33-35$ & $195.83-195.85$ & A & & & & & & & & & \\
\hline $3, \mathrm{CC}(18-20)$ & $200.48-200.50$ & A & & & & & & & & & \\
\hline $4-4,50-52$ & $205.50-205.52$ & A & A & & & & & & & & \\
\hline $4, C C(18-20)$ & $209.98-210.00$ & A & & & C & & & & & & \\
\hline $5-3,34-36$ & $213.34-213.36$ & $\mathrm{~A}$ & A & & & & & & & & \\
\hline $5, \mathrm{CC}(18-20)$ & $219.48-219.50$ & A & & & & & A & & & & \\
\hline $6-3,28-30$ & $222.78-222.80$ & A & A & & & & & & & & \\
\hline $6, \mathrm{CC}(18-20)$ & $228.98-229.00$ & A & A & & & & & & & & \\
\hline $7-3,80-82$ & $232.80-232.82$ & A & & & & & A & & & & \\
\hline $7, \mathrm{CC}(18-20)$ & $238.48-238.50$ & A & A & & & & A & & & & \\
\hline $8-3,60-62$ & $242.10-242.12$ & A & A & & & & & & & & \\
\hline $8, C C(18-20)$ & $247.98-248.00$ & A & A & & & & & & & & One large, rounded, basalt pebble \\
\hline $9-2,60-62$ & $250.10-250.12$ & $\mathrm{C}$ & $\mathrm{F}$ & & & & & & & & \\
\hline $9, \mathrm{CC}(18-20)$ & $257.48-257.50$ & $\mathrm{C}$ & $\mathrm{F}$ & & & & & & & & 2 rounded basalt, 1 angular andesite pebble \\
\hline $10-2,90-92$ & $259.90-259.92$ & & $\mathrm{~F}$ & $\mathbf{P}$ & & & & & & & \\
\hline $10, \mathrm{CC}(18-20)$ & $266.98-267.00$ & & $\mathrm{~F}$ & $\mathbf{P}$ & & & & & & & \\
\hline $11-1,26-28$ & $267.26-267.28$ & & C & $\mathbf{R}$ & & & & & & & \\
\hline
\end{tabular}

${ }^{a}$ Abundance: $\mathrm{P}=$ present, $\mathrm{R}=$ rare, $\mathrm{F}=$ few, $\mathrm{C}=$ common, $\mathrm{A}=$ abundant.

b Preservation: $\mathbf{G}=$ good, $\mathrm{M}=$ moderate, $\mathrm{P}=$ poor, $\mathrm{R}=$ replaced.

c Adjusted sub-bottom depth. 